\title{
El control político de la retaguardia republicana durante la Guerra Civil. Los tribunales populares de justicia
}

\author{
Glicerio Sánchez Recio \\ Universidad de Alicante
}

La rebelión militar de julio de 1936 no consiguió los objetivos completos que se habia marcado, la ocupación del poder del Estado y el control de todo el territorio nacional; pero se ha de reconocer que obtuvo éxitos parciales importantes, que triunfó en determinadas regiones y provincias, en las que pudo organizar un incipiente gobierno y desde las que lanzó la ofensiva contra aquéllas que se mantuvieron leales a la República. Así pues, el fracaso parcial de la rebelión militar condujo a la sociedad española a la guerra civil.

\section{LA REPRESIÓN INCONTROLADA DE LAS PRIMERAS SEMANAS}

La rebelión militar fue otra de las manifestaciones, la más peligrosa sin duda, de la profunda división que existía en la sociedad española y, una vez producida, no sólo acentuó la división y el antagonismo sino que agrandó el hondo sentimiento de violencia que se hizo patente tanto en los frentes de combate como en las respectivas retaguardias. Ante esto, no es una cuestión vana preguntarse por la responsabilidad que tuvieron los gobiernos o centros de poder de ambos bandos enfrentados en los acontecimientos de ese orden acaecidos en cada una de las zonas.

En la zona sometida a los militares rebeldes se implantó, al menos en lo que se refiere al orden público, un fuerte control centralizado de acuerdo con lo establecido en los bandos de declaración de guerra ${ }^{1}$, aunque es

Ver alguno de esos bandos en: Diaz PLAJA, F.: La guerra de España en sus documentos; págs. 14-16 y 36-38. Barcelona, 1970. En ellos se aplicaba lo establecido por la ley de Orden Público de 28 de julio de 1933 en el caso de declaración del estado de guerra, articulos 48 y ss. (Gaceta de Madrid del día 30) 
preciso reconocer un cierto consentimiento para que determinadas organizaciones efectuaran, al margen de toda ley, una fuerte represión contra los ciudadanos de actitudes firmemente republicanas y militantes o simpatizantes de las formaciones y los programas de la izquierda. Las organizaciones represoras pertenecian a las formaciones políticas de la extrema derecha que se habian adherido y apoyado la rebelión e, incluso, habían colaborado antes con representantes de los militares rebeldes para crear la trama de la rebelión. Con ello no se hacía otra cosa que cumplir las instrucciones del Director -el General Mola- para las horas siguientes a la insurrección ${ }^{2}$. De esta forma, las organizaciones represoras, entre las que destacaban las de Falange Española, se convertían en excelentes auxiliares de los militares rebeldes ${ }^{3}$.

Pero la rebelión militar, aparte de dividir el territorio nacional, asestó un duro golpe al Estado republicano de forma que no sólo paralizó su actividad sino que, más aún, desintegró su organización. Al quedar anulada la autoridad central del Estado, surgieron otras, tanto en las regiones históricas, tuvieran o no estatuto de autonomía, como en aquéllas en las que el sentimiento nacionalista presentaba un menor calado. El gobierno republicano no había desaparecido pero no se reconocía su autoridad; podría decirse que ésta no llegaba más allá de las tapas del diario oficial en el que se publicaban sus disposiciones.

La atomización del poder se acentuó más aún a través del protagonismo que desempeñaron las organizaciones políticas y sindicales de la izquierda republicana, que antepusieron sus objetivos programáticos y estratégicos a los del ejercicio sistemático y efectivo de la autoridad, que debería haber intentado prioritariamente utilizar todos los medios para neutralizar a los rebeldes y ganar la guerra civil.

En aquellas circunstancias, organizaciones revolucionarias, grupos de incontrolados e, incluso, representantes de las autoridades republicanas intervinieron con asiduidad para controlar políticamente a la población en la retaguardia. Con este motivo, y de forma generalmente arbitraria, tuvieron lugar detenciones, asesinatos ("paseos"), persecuciones y requisas de bienes contra aquéllos que eran manifiestamente partidarios de los rebeldes, simpatizantes y, en algunos casos, simples votantes de las

Ver al respecto: ARostegul, J.: “Conspiración contra la República», en La guerra civil, n³; págs. 6-40. Edit. Historia 16. Madrid, 1986.

Ver: Fraser, R.: Recuérdalo tú y recuérdalo a otros. Historia oral de la guerra civil españo1a. Barcelona, 1979. En el volumen I, págs. 225-231, pueden verse varios testimonios y actitudes en torno a esta cuestión procedentes de militantes falangistas, carlistas y oficiales del ejército. 
organizaciones de la derecha. Para llevar a cabo esa labor de control político y depuración se crearon tribunales revolucionarios que tomaron distintas denominaciones, según se hallaran representantes de organizaciones diversas -Comité de justicia del Frente Popular- o estuvieran formados por miembros de una sola organización -Comité de Salud pública o Comité revolucionario-. En algún caso estos tribunales revolucionarios actuaron con el reconocimiento de las autoridades regionales, como sucedió con las oficinas juridicas de Barcelona y otras ciudades catalanas, cuyo funcionamiento fue regulado por la Consellería de Justicia de la Generalitat, y a las que se asignaron los objetivos de asesorar a las organizaciones obreras y a los particulares y revisar los procesos penales de carácter social que habian tenido lugar en Cataluña antes de la rebelión militar ${ }^{4}$; y el gobierno regional de Asturias y León, por su parte, se anticipó al gobierno de Madrid en la constitución de un tribunal popular para hacerse cargo de los procesos abiertos contra las personas incursas en el delito de rebelión militar ${ }^{5}$.

La incapacidad del gobierno central, la atomización del poder y la arbitrariedad de las organizaciones revolucionarias facilitaron los avances rápidos del ejército rebelde, que lograba unir en pocas semanas el núcleo antirrepublicano andaluz con el de la Submeseta Norte a lo largo de la frontera portuguesa y de las provincias extremeñas. Alterar la dirección de la guerra requería, en primer lugar, reformar el gobierno central, lo que suponía asimismo la colaboración de todas las organizaciones republicanas y de la izquierda, tanto las políticas como las sindicales; y precisaba, después, terminar con los procedimientos represivos ya descritos para ganar la confianza de la población y obtener la credibilidad internacional. Para llevar a cabo estas operaciones era necesaria la participación de todas las fuerzas republicanas y revolucionarias ya que el objetivo no era sólo la formación de un gobierno de unidad sino que se trataba de una auténtica reconstrucción del Estado.

Un claro anticipo de la operación que se preparaba -pacto entre el gobierno republicano y las organizaciones de la izquierda- fue la publicación de los decretos del 23 y 25 de agosto de 1936 por los que se creaban, en

\footnotetext{
4 Ver: BARRIOBERo y HeRRAN, E.: Un tribunal revolucionario. Cuenta rendida por el que fue su presidente. Barcelona, 1937; NIETO, J.: Cómo actuó en Badalona la justicia revolucionaria. Badalona, s.a. y PAGES I BLANCH, P : “La administración de justicia en Cataluña durante la guerra civil española (1936-1939)", en Justicia en Guerra, págs. 47-63. Edit. Ministerio de Cultura. Madrid, 1990.

5 A.H.N.: Causa General de Oviedo, Leg. 1344. Ver la pieza especial sobre la Actuación del Consejo General de Asturias y León.
} 
Madrid y en el resto de las provincias leales respectivamente, tribunales especiales para juzgar los delitos de rebelión, sedición y los cometidos contra la seguridad exterior del Estado ${ }^{6}$. En las mismas fechas, la Generalitat de Cataluña publicaba unos decretos con idéntica finalidad, adoptando un proceso paralelo e independiente del seguido por el gobierno de la República, y que se mantendría hasta finales de abril de $1937^{7}$. Unos días más tarde, a principios de septiembre, se formaba el primer gobierno de Largo Caballero ${ }^{8}$, pero el gran pacto culminó dos meses después, cuando entraron en el gobierno de la República los representantes de la CNT. Por lo tanto, a partir de los primeros días de noviembre, el gobierno de la República había recuperado todo el poder del Estado, y podía dedicarse preferentemente a contener el avance de los rebeldes y ganar la guerra, para lo que necesitaba también reorganizar la retaguardia ${ }^{9}$. En este contexto histórico y dentro de aquella estrategia política es donde hay que situar el completo desarrollo y la aplicación de la justicia popular.

\section{EL ENTRAMADO DE LA JUSTICIA POPULAR}

Así pues, por los citados decretos del 23 y 25 de agosto de 1936 se creaban los tribunales especiales contra la rebelión, la sedición y los delitos cometidos contra la seguridad exterior del Estado, y a través de ellos el Estado republicano recuperaba efectivamente su poder judicial. Esta justicia adoptó el calificativo de «popular», que fue sancionado por la ley del 7

\footnotetext{
- Ver estos decretos en Gaceta de la República, 24 y 26 de agosto de 1936. La legislación relativa a la justicia popular puede consultarse en MONLEON DE LA LLUVIA, A.E.: Catorce meses de legislación revolucionaria. Valencia, 1937. Ver asimismo: SANCHEZ RECIO, G.: Justicia y guerra en España. Los tribunales populares (1936-1939). Edit. Instituto de Cultura "Juan Gil-Albert". Alicante, 1991.

Sobre esta cuestión ver las obras: RuBio + TudurI, M.: La Justicia a Catalunya. 19 juliol 1936 - 19 febrer 1937. Barcelona, 1937; y PAGES I BLANCH, P.: Op. cit.

8 En este gobierno estaban representados socialistas, ugetistas, comunistas, republicanos y nacionalistas (PNV y ERC).

9 Las medidas más representativas en este sentido son los decretos sobre el control y la regulación de las actividades económicas, tanto los del Gobierno de la República como los de la Generalitat de Catalunya, y los relativos a la constitución de los consejos provinciales y locales, publicados todos ellos entre los meses de septiembre y diciembre de 1936. Ver a este respecto las obras: BrICALL, J.M.: Politica económica de la Generalitat (1936-1939): Evolució i formes de producció industrial. Barcelona, 1970; "La economía española (1936-1939)", en La guerra civil española . 50 años después; págs. 361-417. Barcelona, 1985; SANTACreu Soler, J.M.: Cambio económico y conflicto bélico. Transformaciones económicas en la retaguardia republicana (Alicante 1936-1939). Tesis doctoral (editada en microfichas). Universidad de Alicante, 1988; L'economia valenciana durant la guerra civil. (Protagonisme industrial i estancament agrari). Edit. IVEI. València, 1992; y Girona AlBuixec, A.: Guerra y revolució al Pais Valencià, págs. 38-47. Valencia, 1986.
} 
de mayo de $1937^{10}$. Este término tenía una gran resonancia entre la población leal a la República, pero los tribunales no eran estrictamente populares sino que estaban formados por tres magistrados -sección de derecho- y un jurado popular -jueces de hecho- de distinta composición numérica según las etapas. Sin embargo, los jueces de hecho tenían grandes facultades acerca de la aceptación de las pruebas, el desarrollo de la vista pública de las causas, la calificación de los hechos y la revisión de las sentencias. Es decir, que sus facultades eran mucho más amplias que las que habia tenido habitualmente el jurado popular en España ${ }^{11}$.

El jurado popular estaba formado por representantes de los partidos afectos al Frente Popular y por los de las Centrales Sindicales afines a éste; es decir, se entendía por pueblo no el conjunto de los ciudadanos sino sólo aquéllos que pertenecían o se identificaban con las organizaciones que se mantenían leales y defendian a la República. Por lo tanto, a través del jurado las organizaciones revolucionarias y los partidos republicanos y de la izquierda se incorporaban a la administración de la justicia que se impartía desde el Estado; de este modo, la justicia popular adquiría su categoría esencial de política y se convertía en uno de los pilares defensivos de la República. Por ello, las actuaciones de los tribunales populares en la retaguardia se comparaban con las que efectuaban los milicianos en el frente de batalla ${ }^{12}$.

Aparte de los elementos referentes a la composición y a las competencias de cada una de las secciones de los tribunales populares, los citados decretos asignaban a dichos organismos judiciales instrumentos y mecanismos propios de jurisdicción especial, que sólo adquiría antes carácter general en los casos de declaración de estados de alarma y de guerra, de acuerdo con la ley de orden público. Se trataba del procedimiento sumarísimo ${ }^{13}$ y de la aplicación del Código de Justicia Militar. Pero esto no quiere decir que se aplicara pura y simplemente la jurisdicción militar, sino que la justicia de excepción ${ }^{14}$ de la República incorporaba esos elementos

10 Ley de compilación penal, en Gaceta de la República, 13 de mayo de 1937.

11 Ver AlejandRE, J.A.: La justicia popular en España. Análisis de una experiencia histórica: los tribunales de jurados, pág. 168. Madrid, 1981.

12 A esto se refieren repetidamente los presidentes de los tribunales en sus intervenciones dirigidas al público asistente a los juicios. Ver como ejemplo lo publicado en El Socialista, Madrid, 18 de septiembre de 1936 .

${ }_{13}$ Se entiende por procedimiento sumarisimo aquél al que se somete una causa judicial, civil o criminal, y que por la urgencia o senciliez del caso litigioso, o por la gravedad o flagrancia del hecho criminal, señala la ley una tramitación brevisima (Dicc. R.A.).

14 La justicia popular de la República ha sido calificada tambien de excepción debido a las circunstancias en que se creó y a los fines que le fueron asignados. 
procedentes de la jurisdicción militar sin que fuera preciso para ello la declaración del estado de guerra ${ }^{15}$. Más aún, cuando en el verano de 1938 se presentó en el Consejo de Ministros el proyecto de militarización de los tribunales de justicia, se produjo una profunda crisis política ${ }^{16}$, problema que se habría evitado con la previa declaración del estado de guerra.

Los jurados de urgencia fueron otros organismos judiciales, creados en octubre de 1936, que tenian por objeto juzgar los casos de desafección, es decir, hechos, sentimientos y actitudes de difícil precisión y que, por sí mismos, no constituían delitos. Formados por un juez y dos representantes populares, y habida cuenta de la especificidad de los casos de su competencia, tendieron a imponer penas y sanciones económicas leves, por lo que las autoridades judiciales aconsejaron precaución y mayor dureza al juzgar este tipo de actos ${ }^{17}$.

Los jurados de guardia fueron creados únicamente en Madrid en las mismas fechas y tenian por competencia el conocimiento de hechos relacionados con el orden público: propaganda a favor de los rebeldes, acaparamiento de víveres o manipulación de los precios, y actos relacionados con el sabotaje y el terrorismo. La función de estos organismos judiciales consistía en destinar a los acusados a los tribunales populares competentes, una vez precisada la naturaleza de los delitos.

La acción penal de los tribunales populares se completaba con la del Tribunal de Responsabilidades Civiles, creado también por sendos decretos de los meses de septiembre y octubre de 1936, y cuya función consistía en imponer a los sentenciados por los tribunales de lo penal las sanciones económicas con las que debian reparar los daños ocasionados al Estado por las actuaciones de rebeldía y desafección. Pero con la

t5 El Gobierno de la República no declaró el "estado de guerra" en todo el territorio leal hasta el 9 de enero de 1939.

16 Ver Tuñón DE LARA, M.: «La crisis de agosto y las Cortes de San Cugat", en La guerra civil. Historia 16. Vol. 21, págs. 108-114. Madrid, 1986.

17 Ver la opinión del fiscal de la Audiencia Provincial de Castellón sobre las actuaciones de aquel tribunal durante el año 1937: "La naturaleza de los hechos delictivos que constituyen la naturaleza propia de este tribunal, exige, para juzgar con el mayor acierto posible, una aguda observación por parte del jurado, respecto a todas las circunstancias del hecho, antecedentes del acusado y conducta y relaciones del mismo. Las pruebas reales y objetivas de su delincuencia, no están, muchas veces, tanto en lo que aparece en los autos como pruebas documentales y testificales directas, como en aquellos otros elementos de juicio sacados de las palpitaciones sentidas en el ambiente social y que constituyen muchas veces el medio de llegar a descubrir la verdad. Creo que este Jurado de Urgencia, lejos de obrar bajo tal criterio, se ha dejado llevar de su espiritu excesivamente legalista...", en Memoria de la Fiscalia, 1937. A.H.N. Sección Guerra Civil de Salamanca. Scc. Castellón P.S., Leg. 11. 
intervención de este tribunal, además de completarse la acción judicial, la administración de la justicia se ponía en relación con los planes de reforma socioeconómica previstos por los gobiernos de la República, ya que el producto de las sanciones judiciales y el de las incautaciones, a las que el tribunal daba también formalidad jurídica, deberían destinarse a la reparación de los daños ocasionados por la guerra y a mantener o financiar los proyectos de reforma socioeconómica ${ }^{18}$.

Pero esta justicia de excepción, que se había especializado en juzgar los diversos tipos de hechos delictivos y actos relacionados con la rebelión, terminó invadiendo toda la administración de la justicia, primero, por la extensión de su competencia al conocimiento de los delitos comunes (febrero de 1937) y, después, por la integración de los tribunales especiales en las Audiencias Provinciales (mayo y agosto de 1937). A partir de ahí, puede afirmarse con toda propiedad que la justicia popular, una vez alcanzado ese complejo desarrollo, había absorbido la jurisdicción ordinaria $y$, en consecuencia, se habia creado un nuevo modelo de administración judicial. Así pues, la justicia popular superaba su carácter de excepción, y consiguientemente transitorio, y asumia el de estable y permanente, contradiciendo su propio origen.

En el momento en el que la justicia popular se habia convertido, de hecho, en ordinaria, había cumplido también prácticamente la función para la que había sido creada -juzgar los delitos relacionados con la rebelión-; pero en el tramo final de la guerra civil eran otros los hechos delictivos y los actos que exigían la intervención prioritaria de la administración judicial: el espionaje, el derrotismo y la acaparación de bienes de subsistencia. De ahí que para hacerse cargo de ellos se crearan nuevos tribunales: el de espionaje y alta traición (junio de 1937) y los especiales de guardia (noviembre de 1937 y marzo de 1938), que no eran ya propiamente populares y que actuaron con una gran contundencia a causa de lo cual recibieron el título de "tribunales de hierro" con el que sus propios miembros los calificaron ${ }^{19}$. Lo mismo que sucedió con los tribunales populares, el de espionaje y alta traición y los especiales de guardia, a partir de esas últimas fechas, se ocuparon también de hechos delictivos

\footnotetext{
18 Para administrar el producto de las sanciones e incautaciones el Ministerio de Hacienda creó la Caja General de Reparaciones. Ver SÁnCHEz Recio, G.: La República contra los rebeldes y los desafectos. La represión económica durante la guerra civil. Edit. Secret. de Public. Universidad de Alicante, 1991.

19 Ver SÁNCHEZ RECIO, G.: Justicia y guerra en España. Los tribunales populares (1936-1939). Op. cit., págs. 166-175.
} 
tipificados en los códigos de justicia y de otros actos que no tenían tal categoría, como los de derrotismo. Por lo tanto, en la etapa final de la guerra estos últimos tribunales fueron los que efectuaron la mayor parte de las actuaciones judiciales y a través de los cuales se ejerció la represión más dura en la retaguardia ${ }^{20}$.

\section{OTRAS MEDIDAS DE CONTROL POLITICO: REGISTROS Y DETENCIONES}

El gobierno de la República, aparte de la creación y puesta en funcionamiento de los tribunales populares, publicó también otras normas relativas a los registros domiciliarios y las detenciones, con el ánimo de controlar las actividades que hasta entonces habian desarrollado las organizaciones revolucionarias y devolver la tranquilidad a la población.

Esas medidas fueron tomadas por sendas órdenes del Ministerio de la Gobernación, los dias 6 y 9 de octubre de $1936{ }^{21}$, cuando el gobierno de Largo Caballero había tomado ya otras resoluciones destinadas al control de las milicias ${ }^{22}$. En la primera de las órdenes ministeriales, sobre los registros domiciliarios, se decía de forma clara y contundente: "A partir de la fecha de esta orden sólo podrán realizar registros domiciliarios los agentes de la autoridad y las milicias de investigación integradas en las milicias de vigilancia de retaguardia; pero será requisito indispensable que el registro sea ordenado por la dirección general de seguridad..." (art. 3).

Pero esto suponía, en primer lugar, la desaparición de todos los grupos que anteriormente habian actuado en trabajos de investigación, a los que se invitaba a integrarse "en la sección de investigación de las milicias de vigilancia de la retaguardia»; ahora bien, deberian entregar una relación de los miembros de dichos grupos y de sus jefes y los carnés que utilizaban (arts. 1 y 2). Se especificaban, a continuación, las condiciones exigidas

20 Ver como testimonio el párrafo de una carta que el Fiscal General de la República dirigia desde Barcelona a uno de los fiscales de Madrid, a finales de abril de 1938: "Por aquí se trabaja intensamente, hasta ahora en los tribunales de guardia de Barcelona van celebrados 272 juicios, en los cuales han comparecido 888 inculpados, $y$ ha habido noche en la que se han dictado cuarenta y una sentencias de pena capital, así que ya ves que también aqui tenemos para entretenernos", en A.H.N. Secc. Causa General de Madrid, Leg. 816.

21 Ordenes del Ministerio de la Gobernación sobre "Registros domiciliarios" y "Detenciones", en Gaceta de la República, 7 y 11 de octubre de 1936.

22 El decreto sobre la militarización de las milicias, del 30 de septiembre. Ver SALAS LARRAZABBAL, R.: Historia del ejército popular de la Republica. Vol. I, págs. 499-500. 
para efectuar un registro y se referian los objetos que podían incautarse, las armas y municiones y todo cuanto tenga "el carácter de ofensivo o defensivo", que pueda ser susceptible "racionalmente" de ser utilizado contra el régimen republicano (art. 4), y las subsistencias y ropas, si hay sintomas de acaparación y pueden ser útiles a los hospitales y a las milicias; pero no se incautarán "muebles, efectos, valores, ropa, etc." si no hay orden expresa, por escrito, de la Dirección General de Seguridad (art. 5). Finalmente, se establecía, en el artículo 6 , que quienes realizaran registros sin la debida autorización o no cumplieran los requisitos marcados, serian "detenidos y sometidos como enemigos del régimen al tribunal competente".

Respecto a las detenciones, en la orden correspondiente, se decia, en primer lugar, quiénes estaban autorizados a efectuarlas: "las autoridades militares y gubernativas y sus agentes. Se consideran como tales, además de los que tienen ese carácter, los milicianos que poseen carnet de milicias de vigilancia de retaguardia". Si se trata de la comisión de un delito "in fraganti», cualquier ciudadano puede efectuar la detención, entregando al detenido a la autoridad más próxima (art. 1). Los detenidos serán conducidos obligatoriamente «al local que señalará la Dirección General de Seguridad» y no algún otro (art. 2). A las setenta y dos horas de la detención, una vez efectuados los interrogatorios, los detenidos deberán ser puestos en libertad, a disposición del tribunal competente, popular, de urgencia o juez ordinario, o declarar la detención gubernativa (art. 5). Los puestos en libertad podrán ser detenidos «por existir nuevas pruebas sobre las mismas causas", pero para ello será preciso contar con una orden escrita de la Dirección General de Seguridad (art. 6).

Con las anteriores medidas, el gobierno de la República no sólo pretendía controlar unas actividades, en las que habían destacado las organizaciones revolucionarias, sino más aún normalizarlas y, con la aplicación estricta de la ley, ganarse la confianza y transmitir seguridad a los ciudadanos. Sobre esto cabe resaltar tres de las medidas adoptadas por sus repercusiones inmediatas: la disolución de los grupos que, al margen de la autoridad constituida, habían ejercido funciones de vigilancia e investigación, la declaración de enemigos del régimen y la adopción de medidas pertinentes contra los que no se atuvieran a lo establecido por la ley, y la prohibición de llevar a los detenidos a lugares distintos de los ordenados por la Dirección General de Seguridad. En octubre de 1936, por lo tanto, el gobierno tenía más poder para tomar decisiones e imponerlas; anteriormente, sin embargo, cuando las autoridades provinciales y regionales intentaron aplicar la ley, habían sido desoídas. 
Así, en la Hoja oficial extraordinaria del comité ejecutivo popular de Valencia, del día 23 de julio de 1936, se publicó un bando, titulado "Ordenes severas" y firmado por el gobernador civil, que, en su mayor parte, estaba dedicado a prohibir los registros domiciliarios que se hacian al margen de la ley y por agentes no autorizados ${ }^{23}$. Por otra parte, el Gobierno Regional de Asturias nos pone en contacto con otra de las realidades más duras de los primeros meses de la guerra, el asesinato de los presos durante los traslados, ciertos o simulados, de un lugar a otro; de ahí las fatales connotaciones del término "paseo" desde los primeros días de la subversión. En el Boletín Oficial de la Provincia de Oviedo, entre el 15 y el 22 de octubre, se publicaron varias disposiciones referentes al traslado de presos y a las detenciones ilegales ${ }^{24}$. En el número 1, del 15 de octubre, salia un edicto del dia 8 , firmado por Belarmino Tomás como gobernador de la provincia, en el que de forma contundente se advertía a los jefes de prisión la prohibición «de recibir, trasladar o libertar detenidos" sin la orden previa de las autoridades competentes; asi mismo, se anunciaba que las detenciones que se realizaran, sin estar debidamente autorizadas, serían consideradas "como actos facciosos y sus autores entregados al tribunal popular de justicia". En el mismo número del Boletín, se publicaba un decreto del Departamento de Interior y Justicia del Consejo General de Asturias y León, del 7 de septiembre, en el que, además de las anteriores medidas sobre la custodia y traslado de presos, se mandaba otra de gran interés sobre la ejecución de las sentencias dictadas por los tribunales populares, que se habían constituido en Asturias antes de la publicación de los decretos de agosto por el gobierno de la República ${ }^{25}$. Por otra orden del mismo departamento, del 21 de septiembre, publicada en el Boletín, número 7, del 22 de octubre, con el fin de fortalecer la seguridad de los detenidos, se fijaba un horario para efectuar los

23 Entre los dias 22 y 28 de julio, en Valencia, no se publicó prensa diaria. Esta Hoja... se imprimió en los talleres de El Mercantil Valenciano. En un párrafo de dicho bando se expresaba la actitud de la autoridad y su incapacidad para defenderla: “Las milicias populares deberán replegarse inmediatamente a sus respectivos centros $u$ organizaciones y quedar alli concentradas, por ser innecesarios de momento sus servicios en las calles de la ciudad. Unicamente prestarán los servicios que se les ordene por escrito por parte de la autoridad".

${ }_{24}$ Ver en Causa General de Oviedo la citada pieza especial sobre la «Actuación del Consejo General de Asturias y León"; ver tambièn sobre el mismo tema la disposición del Comité Ejecutivo Popular de Valencia en El Mercantil Valenciano del 1 de octubre, pág. 1

25 Se decia en el decreto: "Mientras no queden constituidos los tribunales de justicia, atemperados a la nueva organización provincial, quedarán pendientes de ejecución los fallos dictados por los tribunales populares que vienen actuando, y éstos dejarán en suspenso su actuación, remitiendo a este Departamento los expedientes en curso de tramitación". Ver Causa General de Oviedo, pieza y legajo citados. 
traslados «de reclusos no comunes de prisión a prisión» desde las ocho a las diecisiete horas de cada día; sin embargo, los jefes o encargados de las prisiones podrán recibir en éstas, a cualquier hora, a detenidos, siempre que no procedan de otra prisión. Finalmente, por otra orden de la Consejería de Justicia, publicada en el Boletín, número 72, del 12 de febrero de 1937, aparte de insistir en el mismo asunto, se establecía el horario de trabajo y el tipo de presos que podía realizarlo ${ }^{26}$.

\section{LOS RESULTADOS DE LA JUSTICIA POPULAR}

No se pretende aquí tratar solamente de los resultados cuantitativos de la aplicación de la justicia popular: el número de personas juzgadas, la distribución en razón de los delitos y de las penas impuestas, el montante de las sanciones económicas, etc., sino también valorar la función desempeñada por la nueva administración judicial dentro de la situación social y política existente en la España republicana durante la guerra civil.

La justicia popular sirvió de instrumento, como se ha indicado más arriba, para someter bajo la autoridad del Estado a las organizaciones políticas y revolucionarias que durante las primeras semanas del conflicto habian actuado de forma autónoma y arbitraria, y ocasionado, por lo tanto, muchos desmanes; al mismo tiempo, la justicia popular se convirtió en el símbolo de que el Estado republicano respetaba la legalidad y garantizaba los derechos de todos los ciudadanos, incluso los de aquéllos que se habian revelado o que le eran desafectos. Sin embargo, el Estado, para conseguir esos objetivos, había tenido que asumir algunos planteamientos de las organizaciones revolucionarias e, incluso, entregarles el poder político.

Pero la justicia popular fue, sobre todo, el instrumento represivo que el Estado republicano utilizó en la retaguardia, habiendo sido éste el campo en el que se alcanzaron los resultados más efectivos. Para ello se dictaron los decretos adecuados y se efectuó la necesaria depuración previa de los funcionarios judiciales ${ }^{27}$, y los abogados afectos al Frente Popular ocuparon la dirección de los Colegios Profesionales para colaborar en la

\footnotetext{
26 Se decia expresamente: "Que los trabajos en que se empleen los reclusos han de verificarse en la localidad en que esté enclavada la prisión, con la debida vigilancia y jamás después de oscurecer, no pudiendo dedicar a ello a los presos comunes ni a los sociales contra los que existan acusaciones de carácter grave». Ibidem., Legajo citado.

27 Ver SANChez RECIO, G: «Depuración y reforma de la administración de la justicia en la provincia de Alicante durante la guerra civil", en Anales de la Universidad de Alicante. Historia Contemporánea, $n^{9} 6$, págs. 127-144. Alicante, 1988
} 
administración de la justicia y controlar el nombramiento de los abogados defensores de oficio ${ }^{28}$. Con estos antecedentes y animados por la presencia de los jurados populares, los tribunales, desde el principio de sus actuaciones, se convirtieron en una maquinaria perfectamente capacitada no sólo para reprimir sino también para propagar y captar a los ciudadanos en favor de la causa republicana.

La actividad de los tribunales populares fue intensa, y muy numerosas las causas vistas y las sentencias dictadas. No creo que sea necesario reproducir aquí los datos relativos a esas actuaciones ${ }^{29}$; pero sí, analizar a grandes rasgos los tipos de penas impuestas. Evidentemente, el número de condenas a muerte dictadas fue alto y las penas de privación prolongada de libertad (entre la cadena perpetua y los doce años y un día), abundantes; sin embargo, no puede dejarse de lado el fuerte porcentaje alcanzado por las sentencias de absolución y los sobreseimientos, de forma conjunta. A título de ejemplo, véase la tabla siguiente, en la que constan los datos correspondientes a esos tres tipos de sentencias de varios tribunales especiales (populares) contra la rebelión:

Sentencias de los tribunales especiales populares, según los tipos de penas, en tantos por ciento

\section{Tribunales}

Albacete

Alicante

Barcelona

Bilbao

Cartagena

Gijón

Málaga

Madrid

Valencia

Procesa
363
648
427
403
351
224
557
566
89

89
Muerte

26,80

23,46

32,79

31,76

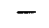

16,32

0,18

8,48

43,82
Reclusión

50,00
16,82
62,06
36,98
15,67
66,52
0,36
30,50
10,11

Absol/Sobres.

10,22

25,11

5,15

29,28

42,73

82,58

24,20

38,20

Por lo tanto, no puede decirse que los tribunales populares aplicaran una justicia política -de excepción- de forma ciega y totalmente predeterminada.

28 Acciones de este tipo tuvieron lugar en Málaga, Valencia, Madrid... En la Causa General (A.H.N.) de esas provincias y en la prensa local hay amplia información sobre los hechos. Respecto a lo acontecido en Cataluña, ver: Rubio i TuduRI: Op. cit., pág. 13.

29 Ver para esto: Sanchez Recio, G.: Justicia y guerra en España..., págs. 124-165. Para los datos de la tabla, pág. 132. 
La intensa dedicación de los tribunales populares consiguió que la mayor parte de los implicados en la rebelión, y en particular los principales protagonistas - militares y civiles- fueran juzgados antes del final de la primavera de 1937, por lo que a partir de esas fechas los tribunales pudieron dedicarse también al conocimiento de los delitos comunes y, poco más tarde, a los casos de desafección, con lo que se abolieron los jurados de urgencia (marzo de 1938), después de la fuerte actividad que habian desarrollado ${ }^{30}$. En el último tramo de la guerra, como ya se dijo, fueron los tribunales especiales de guardia los que impartieron la justicia de la forma más copiosa.

La obra de propaganda y captación efectuada por los tribunales populares tuvo una gran trascendencia, porque las salas en las que se celebraban las vistas públicas de las causas se convirtieron en una especie de escenarios, podría decirse, incluso, que de templos, en los que, además de administrar justicia, se representaban u oficiaban unos actos en los que se execraban los delitos de rebelión y desafección, personificados en los acusados que se condenaban mediante la imposición de la pena o se purificaban con la absolución o el sobreseimiento. Aparte de los acusados, otros actores principales, $u$ oficiantes, eran los miembros del tribunal, en particular el presidente, y el público asistente que se identificaba con los representantes de la República o abjuraba profundamente de los autores de los delitos. Tanto es así que los presidentes de los tribunales juzgaron oportuno intervenir en algunos casos para explicar la presencia de los abogados defensores de oficio en las salas ${ }^{31}$.

Todos estos actos ejercian una fuerte influencia sobre la ciudadanía a través de la publicidad que se les daba en la prensa y en la radio. Creo que nunca en España las actuaciones de los tribunales de justicia han cumplido mejor su doble función de condenar el delito y de corregir la conducta pública mediante la ejemplaridad del castigo. Durante los meses en los que se juzgaron las causas de los principales implicados en la rebelión, en todos los periódicos del país se reservó diariamente una sección

\footnotetext{
30 Las actuaciones mejor conocidas de Jurados de Urgencia son las del de Cartagena y Castellón porque del primero se conserva el listado completo de los encausados (A.H.N. Se. Guerra Civil de Salamanca: Sec. Cartagena P.S., Leg. 48) y del segundo el fichero de las causas y sentencias (A.H.N. Causa General de Castellón, Leg. 1.405,1).

31 El presidente del tribunal popular de Alicante decia en el acto de constitución, refiriéndose a los abogados defensores: "Vienen aqui en turno de oficio a realizar una defensa que tal vez ellos voluntariamente no hubieran aceptado, porque no pueden en manera alguna confraternizar ya que su corazón y su naturaleza rechazan hechos de esta naturaleza...". Ver El Luchador, Alicante, 7 de septiembre de 1936.
} 
dedicada a la justicia popular, con noticias no sólo de los casos y de las sentencias sino también con las declaraciones de los testigos, las intervenciones de los fiscales, y a veces de los defensores, reflejando asimismo el ambiente que se daba en las salas y los sentimientos del público ${ }^{32}$.

A través de los medios de comunicación se transmitía al público la opinión de que por las actuaciones de los tribunales populares la República respetaba los derechos individuales, se eliminaba y aislaba a los enemigos del régimen, se aseguraba la retaguardia y que, en definitiva, se contribuía a ganar la guerra, del mismo modo que lo hacían los soldados y los milicianos en el frente de batalla.

Pero si las actuaciones de los tribunales especiales (populares) ejercieron una gran influencia sobre el público, no sucedió lo mismo con los especiales de guardia -tribunales represores de la última época-, porque los casos que juzgaban no despertaban el menor interés en la sociedad. Menos trascendencia tuvo aún el Tribunal Popular de Responsabilidades Civiles a pesar de la importante función que ejerció en la represión económica, porque sus actuaciones eran exclusivamente de carácter técnico y se efectuaron lejos de la atención pública ${ }^{33}$. Como prueba de la actividad de este tribunal, véase la tabla siguiente ${ }^{34}$ :

Sanciones del T.P.R.C. según la pena impuesta

Pena t.p.

Muerte

Reclus. perpetua

Reclusión menor

Prisión mayor

Prisión menor

Arresto

Absolución

Desconocida

TOTAL
Acusados

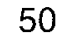

110

99

66

132

26

21

307

811
Sanción

406.750 .000
178.705 .001
51.502 .000
30.680 .000
11.931 .500
945.000
207.001
109.844 .855

799.565 .357
Sanción/acus.
8.135 .000
1.706 .409
520.222
464.848
90.390
36.346
9.857
357.800

32 La fuente principal para tratar esta cuestión, aparte de las referencias que aparecen en la Causa General, es la prensa; pero ésta hay que estudiarla siguiendo los criterios de la importancia de la publicación y de las diversas tendencias ideológicas y programáticas que representaba.

33 Ver Sánchez Recio, G.: La República contra los rebeldes y los desafectos..., págs. 14-16.

34 Para valorar correctamente la actividad de este tribunal, ver: SÁNCHEZ RECIO, G.: Op. cit., págs. 79-113. Las sanciones impuestas por el tribunal se hallan muy lejos del valor contable administrado por la Caja de Reparaciones ya que ésta tenía también bajo su control objetos valiosos y otros bienes (págs. 151-157). 